\title{
Artificial Intelligence: The New Frontier in Surgery
}

\author{
Michael McFarlane
}

\begin{abstract}
This review aims to discuss the advances in artificial intelligence (AI) and the role it now plays in surgery. The discussion outlines the many capabilities of AI in improving the way in which surgery is conducted and a critical review of new AI developments.

Artificial intelligence now well established in several industries has now begun to make a change with significant improvements in the practice of medicine. The use of algorithms that allow advanced computers to have cognitive functions that simulate human thought and actions has given rise to image and speech recognition, and autonomous robots that can perform unsupervised tasks relying on vast databanks of information.
\end{abstract}

A transition from traditional laparoscopic surgery to robotic surgery has already taken place. Artificial intelligence is now beginning to extend the capabilities of surgical robots to encompass autonomy, which will allow them to use information from their surroundings, recognize problems and implement the correct actions without the need for human intervention.

Advances in computing capability, machine engineering and robotics and the ever improving development of smart algorithms is allowing growth of the application of AI at a rapid pace. These developments have resulted in the development of nanorobots that function on a scale of nanometers and have become the next generation system to be integrated with AI and surgery. The use of this technology has resulted in advances in neurosurgery, vascular surgery and oncology.

The future of surgery, like other fields in medicine will be data driven with a significant input from technology. Artificial Intelligence is one advancement that will play a significant role.

Index Terms - artificial intelligence; neural networks; surgery; robotics; nanorobots.

\section{INTRODUCTION}

Artificial intelligence (AI) relies on the use of computer programing to allow algorithms to be used for problem solving and thus endows machines with the ability to take part in decision-making using all available data and to arrive at a diagnosis or course of management which is reliable, accurate and reproducible regardless of the uniqueness of the clinical situation [1]. This usage of devices in medicine has been a natural progression of the benefit of computerization and automation to augment human performance and eliminate fatigue and error [2].

The integration of machines into human endeavour was heralded during the dawn of the Industrial Revolution which allowed the output of human effort to be almost infinitely scaled to previously unimagined proportions. The widespread usage of computers has resulted in even further

Published on July 25, 2020.

M.E.C. McFarlane, Department of Surgery, Radiology, Anaesthetics and Intensive Care University of the West Indies, Mona, Jamaica.

(corresponding e-mail: michaelm500@yahoo.com) growth of productive capacity and has now been applied to the medical sciences, using algorithms common to diagnostic reasoning making the process programmable and universally accessible. Modern healthcare is eminently suitable for AI since over the last decade it has been transformed by the use of digital information storage which has wide application in patient's electronic medical records, medical investigations and advanced image storage and retrieval e.g., picture archiving and communication systems (PACS). Analysis of this data can be done in minute detail for individual patients and compared with a vast amount of data from previous records for immediate decision-making, management planning and long-term prognostication all at the same time. This capability is beyond the skill of a human observer. The smart technology that drives artificial intelligence is based on machine learning (ML), deep learning (DL), artificial neural networks (NL), natural language processing (NLP) and computer vision (CV).

The development of laparoscopic surgery heralded a shift away from traditional surgery by the introduction of technology capable of allowing procedures to be performed using mechanical devices. Up until that point in the history of surgery, procedures were done entirely with the hands with little use of mechanical devices. Since its introduction in the decade of the 80s laparoscopic surgery has made several advances aided by the introduction of robotic surgery. The new precision gained by the use of robots has eliminated errors associated with human intervention such as fatigue and tremor. This has also allowed highly complex procedures to be performed in anatomical sites that were previously thought to be inaccessible.

\section{REVIEW}

\section{A. The Development of Artificial Intelligence}

Improvements in computer processing speed and power coupled with innovative software design has resulted in an ability referred to as "machine learning" which is a significant feature of AI [3]. Previously computers could only be programmed to perform complex tasks related to a specific situation, but these "rules" could not be used to solve another unrelated problem without a completely new program. Machine learning, sometimes called unsupervised learning, together with another concept in computing referred to as "deep learning" now allows the computer to use algorithms with an extensive store of data, to create a new set of "rules" that allows it to adjust, and improve performance in response to feedback similar to cognitive adjustments made by humans [4]. Machine learning and deep learning together seamlessly create artificial neural networks that can adjust output almost instantaneously learning at a speed and combining algorithms to create solutions to problems with more accuracy and reliability 
than humans will be able to. This capability can now be applied to several branches of medicine and surgery [5].

Another advanced AI-powered development that has found wide application in healthcare is computer vision [6]. This technology allows computers to evaluate and interpret the data from images allowing recognition, classification and even modelling of 3-D images. This feature when coupled with deep learning and machine learning has application with medical radiology such as CT and MRI scans and the diagnosis of disease from pathological slides [7], [8]. Intelligent algorithms working in systems with rapidly increasing computer processing power has allowed the development of self-driving cars, and robotics to become commonplace. This ability based on the recognition of images has evolved from tasks such as simple scene and image recognition. The application of technology has allowed real-time computer aided diagnosis and imageguided surgical procedures to be performed. Artificial intelligence has also expanded into the field of human-like recognition of speech. This recognition of language has allowed computers to analyse both spoken and written language identifying scientific words and phrases and even the contextual application, aiding research, derived from the examination of electronic medical records (EMRs). This technology has allowed analysis of large datasets using both linear and non-linear techniques to allow discovery of patterns and associations that would be normally imperceptible to humans [9].

Combining these diverse capabilities of artificial intelligence and the perceived ability of such systems to ultimately design their own newer and more advanced capabilities suggests an ever increasing spiral of the development of this technology to the point of intelligent autonomy. This recruitment of multiple algorithms, referred to as ensemble methodology allows the modern AI systems to use data for prediction, statistical calculation and enhanced flexibility and accuracy in analysing large unrelated data at levels not previously attained without such technology.

\section{B. Application of AI in Surgery}

One of the most futuristic applications of this technology is the development of autonomous robots that could undertake surgical procedures and function independently, making critical decisions and doing complete procedures without the need for human assistance. The introduction of smart technology into the practice of surgery has resulted in the emergence of autonomous or semi-autonomous robots that are capable of performing tasks such as suturing and bowel anastomosis [10]. The best known of these new autonomous systems is the Smart Tissue Autonomous Robot (STAR) that is able to suture and dissect tissue with more precision than a human surgeon can [11]. Using advanced algorithms that can allow the device to have force feedback of tissue density the robot has been used to complete the anastomosis of bowel with satisfactory efficiency and competence. A clear application of this system could be in the field of trauma and emergency surgery where a team on location with just minimal medical training could obtain access and allow the robot to complete the procedure which would normally require the skill of an experienced trained surgeon to perform.

Artificial intelligence has made great strides in laparoscopic robotic surgery allowing access into previously inaccessible anatomical locations as well as being able to perform delicate manoeuvres without susceptibility to fatigue. A surgical robot can pause during a procedure and remain completely still for as long as is required. Also the benefit of applying surgical knowledge previously acquired and programmed allows the algorithms to choose the best surgical approach for each stage of the operation. The use of real time computer vision and video analysis of the procedure allows detailed tracking of the stages of the procedure which can inform the surgeon of steps that may have been missed and which should be revisited. Advanced eye-tracking camera control during laparoscopic surgery, haptic feedback that essential feature that allows the engagement of touch, vibration and resistance that allows the surgeon to recognize and feel the force and resistance for each movement of the robotic arms has made laparoscopic surgery more precise and in many procedures has reduced error significantly [12].

Endoscopic procedures similarly benefit and are significantly improved by AI. Real-time endoscopic image diagnosis allows better detection of polyps and other premalignant lesions during colonoscopy. Advanced robotic platforms using micro-instrumentation and image augmentation has also been of assistance in diagnostic and therapeutic interventions during bronchoscopy [13].

\section{Recent Advances in AI}

Miniature robots have allowed procedures to be done via small incisions. The Heartlander is a mini-robot that traverses the surface of the heart and can be programmed to give small injections to parts of the heart that would normally be inaccessible. It is placed into the chest via a sub-sternal incision and moves to several locations on the surface of the heart [14].

The da Vinci robot system is well established in advanced laparoscopic procedures allowing intricate procedures to be performed with the reduction of human error by eliminating tremor and reducing procedural mistakes which would be impossible without the technology. The da Vinci system allows minimally invasive surgery to be performed more precisely though it still requires the involvement of the surgeon whose hand movements are translated and mirrored by the robotic arms [15].

The most recent advancement in the use of robotics in surgery is the development of nanorobots [16]. These robots are designed to function using nanotechnology on a scale of 1 to 100 nanometers, which are capable of manipulating cell receptors 30 to 50 nanometers in diameter. Nanotechnology coupled with AI is now being applied in the field of neurosurgery using devices that can operate on axons to optimize repair of transected nerves which may aid in the restoration of spinal cord function after spinal cord injury.

Nanotechnology also has other applications in surgery including the ability of these devices to traverse blood vessels to allow treatment to be targeted to almost any location in the body [16]. Uses have emerged in the field of vascular surgery for the treatment of cerebral and peripheral 
aneurysms, and in the field of oncology for the mapping of tumours to facilitate surgical resection.

\section{Imaging}

Diagnostic digital imaging has benefited significantly from the use of AI. Modern CT and MRI images can now be examined and compared with previously archived images to detect subtle changes characterising them as either benign or malignant. This is now being used extensively in thoracic, abdominal and pelvic images and brain imaging. The deep learning algorithms, which are constantly improving, coupled with vast data storage and advanced computing is ideally suited to recognition of abnormalities in medical images. Another area where AI is particularly suited is the analysis of dermatological images for diagnosis. There are now online systems which allow photographs of skin lesions to be uploaded to AI servers with the ability to make a diagnosis almost instantaneously [17].

A recent study comparing the result of using AI for the diagnosis of breast cancer showed that AI was more accurate in detecting more cancers with mammographic features of a breast mass, architectural distortion, and asymmetry than radiologists viewing the same images [18]. Radiologists working with AI assistance had improved specificity and sensitivity in making a diagnosis of breast cancer particularly in patients with dense breasts where the diagnosis is often more difficult.

\section{CONCLUSIONS}

It is anticipated that as these AI systems improve, and the data available to them increases, there will be an exponential improvement, which is expected to take the capability of these systems to a point far beyond human ability. There will be also be a benefit to humans with this human to machine interaction resulting in augmentation of human intelligence to much higher levels. Nanotechnology has ushered in a new era of surgery that can be performed on a scale order of magnitude hitherto not believed possible even with the most advanced systems available today. Coupled with AI this technology will benefit surgery in the field of diagnosis and treatment of a wide range of surgical diseases.

\section{REFERENCES}

[1] Hamet P, Tremblay J. Artificial intelligence in medicine. Metabolism. 2017;69S:S36-S40. doi:10.1016/j.metabol.2017.01.011.

[2] Chen JH, Asch SM. Machine Learning and Prediction in Medicine Beyond the Peak of Inflated Expectations. N Engl J Med. 2017;376(26):2507-2509. doi:10.1056/NEJMp1702071

[3] Deo RC. Machine Learning in Medicine. Circulation. 2015;132(20):1920-1930. doi:10.1161/CIRCULATIONAHA.115.001593.

[4] Esteva A, Robicquet A, Ramsundar B, et al. A guide to deep learning in healthcare. Nat Med. 2019;25(1):24-29. doi:10.1038/s41591-0180316-z.

[5] Cruz JA, Wishart DS. Applications of machine learning in cancer prediction and prognosis. Cancer Inform. 2007;2:59-77. Published 2007 Feb 11.

[6] Voulodimos A, Doulamis N, Doulamis A, Protopapadakis E. Deep Learning for Computer Vision: A Brief Review. Comput Intell Neurosci. 2018;2018:7068349. Published 2018 Feb 1. doi:10.1155/2018/7068349.

[7] Nakata N. Recent technical development of artificial intelligence for diagnostic medical imaging. Jpn J Radiol. 2019;37(2):103-108. doi:10.1007/s11604-018-0804-6
[8] Marchetti MA, Codella NCF, Dusza SW, et al. Results of the 2016 International Skin Imaging Collaboration International Symposium on Biomedical Imaging challenge: Comparison of the accuracy of computer algorithms to dermatologists for the diagnosis of melanoma from dermoscopic images. J Am Acad Dermatol. 2018;78(2):270277.e1. doi:10.1016/j.jaad.2017.08.016.

[9] Wachs JP, Stern HI, Edan Y, et al. A gesture-based tool for sterile browsing of radiology images [published correction appears in $\mathrm{J}$ Am Med Inform Assoc. 2009 May-Jun;16(3):284]. J Am Med Inform Assoc. 2008;15(3):321-323. doi:10.1197/jamia.M241.

[10] Moustris GP, Hiridis SC, Deliparaschos KM, Konstantinidis KM. Evolution of autonomous and semi-autonomous robotic surgical systems: a review of the literature. Int J Med Robot. 2011;7(4):375392. doi:10.1002/rcs.408.

[11] Shademan A, Decker RS, Opfermann JD, Leonard S, Krieger A, Kim PC. Supervised autonomous robotic soft tissue surgery. Sci Transl Med. 2016;8(337):337ra64. doi:10.1126/scitranslmed.aad9398.

[12] Roh HF, Nam SH, Kim JM. Robot-assisted laparoscopic surgery versus conventional laparoscopic surgery in randomized controlled trials: A systematic review and meta-analysis. PLoS One. 2018;13(1):e0191628. Published 2018 Jan 23. doi:10.1371/journal.pone.0191628.

[13] Kudo SE, Mori Y, Misawa M, et al. Artificial intelligence and colonoscopy: Current status and future perspectives. Dig Endosc. 2019;31(4):363-371. doi:10.1111/den.13340.

[14] Patronik NA, Ota T, Zenati MA, Riviere CN. A Miniature Mobile Robot for Navigation and Positioning on the Beating Heart. IEEE Trans Robot. 2009;25(5):1109-1124. doi:10.1109/tro.2009.2027375.

[15] Leal Ghezzi T, Campos Corleta O. 30 Years of Robotic Surgery. World J Surg. 2016;40(10):2550-2557. doi:10.1007/s00268-0163543-9.

[16] Saadeh Y, Vyas D. Nanorobotic Applications in Medicine: Current Proposals and Designs. Am J Robot Surg. 2014;1(1):4-11. doi:10.1166/ajrs.2014.1010.

[17] Li CX, Shen CB, Xue K, et al. Artificial intelligence in dermatology: past, present, and future. Chin Med J (Engl). 2019;132(17):20172020. doi:10.1097/CM9.0000000000000372.

[18] Rodriguez-Ruiz A, Lång K, Gubern-Merida A, et al. Can we reduce the workload of mammographic screening by automatic identification of normal exams with artificial intelligence? A feasibility study. Eur Radiol. 2019;29(9):4825-4832. doi:10.1007/s00330-019-06186-9. 